\title{
LA TRADIZIONE MANOSCRITTA DELLA VITA S. PAULI PRIMI EREMITAE E LE PIÙ ANTICHE EDIZIONI STAMPATE DI QUESTA OPERA GERONIMIANA
}

L'articolo intende presentare, nella sua prima parte, il risultato delle nostre ricerche riguardanti la tradizione manoscritta della Vita S. Pauli monachi Thebaei di san Girolamo e, in particolare, ciò che stabilimmo continuando il lavoro inziato da John Frank Cherf O.S.B.

Inoltre, l'articolo, nella sua seconda parte, analizzerà le più antiche edizioni stampate dell'opera geronimiana presa in considerazione.

\section{LA TRADIZIONE MANOSCRITTA \\ DELLA VITA S. PAULI PRIMI EREMITAE DI SAN GIROLAMO}

Per avvicinarsi al testo stilato da san Girolamo, consultammo i manoscritti rimasti della Vita ${ }^{1}$ : 144 testi contenuti in 140 codici manoscritti ${ }^{2}$ (tutti i manoscritti fino alla fine dell'XI secolo, e i manosciritti più tardivi soltanto occasionalmente). Per quanto riguarda le famiglie manoscritte "A", "D", "E",

${ }^{*}$ Prof. dr. hab. Bazyli Degórski, O.S.P.P.E. - professore di patrologia e di teologia dogmatica dell'età patristica presso la Pontificia Università S. Tommaso d'Aquino a Roma, e professore di patrologia e di antropologia patristica alla Pontificia Facoltà Teologica "Teresianum" a Roma; procuratore generale presso la Santa Sede; email: osppe.roma@gmail.com.

${ }^{1}$ Cf. R. Degórski, Edizione critica della "Vita Sancti Pauli Primi Eremitae" di Girolamo, Institutum Patristicum "Augustinianum", Roma 1987, passim. Cf. anche idem, Stan badań nad "Vita S. Pauli Primi Eremitae" św. Hieronima, in: Archivum Ordinis Sancti Pauli Primi Eremitae I: Dissertationes, vol. 5, ed. S. Świdziński Coesfeld 2010, 161-222; idem, Untersuchungen zu den Handschriften und Drucken über die "Vita S. Pauli Primi Eremitae" vom hl. Hieronymus, in: Ideologie des Paulinermönchtums Ende 15./ Anfang 16. Jahrhundert. Internationales Seminar im ehemaligen Paulinerkloster (1377-1786) Marianka bei Bratislava, vom 11.-15./16 Oktober 2010, Archivum Ordinis Sancti Pauli Primi Eremitae I: Dissertationes, vol. 6/1, ed. L. Weinrich - S. Świdziński, Coesfeld 2012, 8-15.

${ }^{2}$ Per quanto riguarda l'elenco di tutti i codici manoscritti da noi consultati e studiati, cf. Degórski, Edizione critica della "Vita Sancti Pauli Primi Eremitae” di Girolamo, p. 10-27; idem, Przekaz łacińskich rękopisów „,Vita S. Pauli Primi Eremitae” św. Hieronima, Lublin 2000, 67-84. Per quanto concerne, invece, l'elenco generale dei manoscritti della Vita Pauli, cf. Studies in the Text Tradition of. St. Jerome's “Vitae Patrum”, ed. W.A. Oldfather, Urbana 1943, 17-35. 
"R", "K", "L", "P", stabilite da Cherf3, le nostre ricerche non portarono delle novità. Scoprimmo unicamente delle sviste o degli errori, e descrivemmo più dettagliatamente alcuni codici, o facemmo degli emendamenti ${ }^{4}$. Rilevammo, invece, delle più importanti e grandi aggiunte riguardo alle restanti famiglie, ovverossia: "I", "J", "Q", "W", "Z". Inoltre, creammo una nuova famiglia manoscritta: la famiglia " $S$ ", del tutto sconosciuta a Cherf e, in più, giudicammo il manoscritto Vaticanus Lat. 1188 vicino alle famiglie "Q" e "W" e, come tale, manoscritto che le unisce e congiunge.

1. La famiglia "I"'. Ai nove manoscritti, annoverati da Cherf dal gruppo "I", aggiungemmo altri diciasette codici. La famiglia "I" non possiede varianti che non si troverebbero negli altri manoscritti. Ciò nonostante, $\mathrm{i}$ codici di questo gruppo sono più strettamente collegati tra di loro che con qualsiasi altro gruppo, il che ci autorizzò ad unirli. Per quanto riguarda collegamenti più stretti entro il gruppo "I", constatammo che cinque manoscritti di esso sono più vicini reciprocamente 7 . Il Casanatensis Codex 726 e il Vaticanus Urbinas Lat. 1503 si avvicinano al Casinensis 463. Tuttavia, non provengono direttamente da esso, e il Casanatensis Codex 726 non proviene direttamente dal Vaticanus Urbinas Lat. $1503^{8}$. Il Casanatensis Codex 726 e il Vaticanus Urbinas Lat. 1503 non sono copiati direttamente dal Casinensis 463, ma da un manoscritto, o da manoscritti, derivante dal Casinensis $463^{9}$.

Il Casinensis 149 e il Casinensis 140 non corrispondono tra di loro soltanto tre volte ${ }^{10}$. I codici, invece, Sanctae Mariae Maioris 1, nel quale si trovano ben due testi della Vita Pauli, e il Vallicellanus Tomus V condividono con il Vaticanus Lat. 1195 addirittura venticinque varianti. Ciò basta per mostrare che questi due testi, che si trovano nel Sanctae Mariae Maioris 1 e nel Vallicellanus Tomus V, sono strettamente collegati con il Vaticanus Lat. $1195^{11}$. Il Vallicellanus Tomus V, però, non deriva direttamente dal Vaticanus Lat. 1195, perché possiede anche delle proprie lezioni ${ }^{12}$.

I due testi della Vita Pauli, contenuti nel Sanctae Mariae Maioris 1, non furono copiati direttamente dal Vaticanus Lat. 1195, bensì da un manoscritto, a noi

${ }^{3}$ Cf. J.F. Cherf, The Latin Manuscript Tradition of the "Vita S. Pauli", in: Studies in the Text Tradition of. St. Jerome's “Vitae Patrum”, p. 65-142.

${ }^{4}$ Cf. R. Degórski, Edizione critica della "Vita Sancti Pauli Primi Eremitae" di San Girolamo, Institutum Patristicum “Augustinianum”, I-VI, Roma 1987 (dattiloscritto), 1278-1309, 1368-1390.

5 A proposito di questa famiglia cf. idem, La famiglia "I" della tradizione manoscritta della

"Vita S. Pauli Primi Eremitae" di San Girolamo, DPa 5 (1992) 5-24.

${ }^{6}$ Cf. Cherf, The Latin Manuscript Tradition, p. 87-94.

${ }^{7}$ Cf. Degórski, Przekaz łacińskich rękopisów, p. 140-141.

${ }^{8}$ Cf. ibidem, p. 141-144.

${ }^{9}$ Cf. ibidem, p. 144.

${ }^{10}$ Cf. ibidem, p. 144-145.

${ }^{11}$ Cf. ibidem, p. 145-148.

${ }^{12}$ Cf. ibidem, p. 149-151. 
sconosciuto, derivante dal Vaticanus Lat. 1195. Pare che i testi del codice Sanctae Mariae Maioris 1 fossero copiati dallo stesso scrivano (oppure che fossero prodotti dello stesso scriptorium), il quale si servisse di un testo comune. Quindi, possiamo considerare questi testi del Sanctae Mariae Maioris 1 due "gemelli"13.

Il Neapolitanus Lat. VIII B 3 e il Vaticanus Barberinianus Lat. 702 sono collegati con il Vindobonensis Lat. 994 e il Basilicanus D. 190, che appartengono anche alla famiglia "I". Il Vindobonensis Lat. 994, però, è più vicino al Neapolitanus Lat. VIII B 3 che al Vaticanus Barberinianus Lat. $702^{14}$.

Il Basilicanus D. 190 è più strettamente unito al Vaticanus Barberinianus Lat. 702, al Vindobonensis Lat. 994 e al Neapolitanus Lat. VIII B 3. Ciò nonostante, non proviene direttamente da nessuno di essi, dal momento che ha delle varianti che a volte si incontrano nel Vaticanus Barberinianus Lat. 702, e a volte ha delle varianti presentate dal Vindobonensis Lat. 994 e dal Neapolitanus Lat. VIII B 3, oppure presenta anche una lezione propria ed indipendente da essi ${ }^{15}$.

Il Vaticanus Lat. 344, il Vaticanus Palatinus Lat. 860, e i codici Vaticanus Lat. 358, 363, 349, 5087, 5119, nonché il Iesuiticus 973, il Vallicellianus Codex C. 74, il Casanatensis Codex 253 e l'Angelicus 234 formano un'unità più stretta e derivano da un comune antenato e formano, in tal modo, uno dei sottogruppi della famiglia "I"16. I manoscritti Vaticanus Lat. 358 e 363, invece, furono copiati dallo stesso codice e differiscono tra di loro soltanto in pochi punti. Tuttavia, l'uno non è la copia diretta dell'altro: essi sono "gemelli"17.

2. La famiglia "J". Ai sette manoscritti, annoverati da Cherf al gruppo "J"18, aggiungemmo il Vaticanus Lat. 362 (del 1459). Il Parisinus Lat. 16736 e il Parisinus Lat. 17003 non presentano alcuna differenza (neanche secondaria), e il Parisinus Lat. 17003 fu copiato dal Parisinus Lat. $16736^{19}$.

Esiste una più stretta dipendenza, da una parte, tra il Bernensis Lat. 199 e il Remensis 1402 e, dall'altra parte, tra il Vaticanus Reginensis Lat. 589 e tra i manoscritti posteriori. Il Vaticanus Reginensis Lat. 589 e il Bernensis Lat. 199, però, presentano solo un'unica variante comune assente negli altri manoscritti (eccezion fatta per il Remensis 1402), mentre ci sono tra di essi circa venti differenze. Il Vaticanus Reginensis Lat. 589 non deriva, quindi, direttamente dal Bernensis Lat. $199^{20}$.

L'Escurialensis a II 10, l'Andegavensis 154, il Parisinus Lat. 16736 e il Vaticanus Lat. 362 non derivano dal Vaticanus Reginensis Lat. 589, perché

\footnotetext{
${ }^{13}$ Cf. ibidem, p. 151-153.

${ }^{14}$ Cf. ibidem, p. 153-157.

${ }^{15}$ Cf. ibidem, p. 157-159.

${ }^{16}$ Cf. ibidem, p. 159-164.

${ }^{17}$ Cf. ibidem, p. 164-167.

${ }^{18}$ Cf. Cherf, The Latin Manuscript Tradition, p. 94-96.

${ }^{19}$ Cf. Degórski, Przekaz łacińskich rękopisów, p. 178.

${ }^{20}$ Cf. ibidem, p. 178-180.
} 
non vanno con esso d'accordo in quei punti nei quali non possono esserci dubbi circa la lezione del Vaticanus Reginensis Lat. 589. Essi provengono forse da un codice più antico rispetto al Vaticanus Reginensis Lat. 589, in cui furono apportate tante correzioni. Tali differenze escludono l'ipotesi che l'Escurialensis a II 10, l'Andegavensis 154, il Parisinus Lat. 16736 e il Vaticanus Lat. 362 provengono direttamente da un codice comune ${ }^{21}$.

L'Andegavensis 154, nonostante le somiglianze con l'Escurialensis a II 10 e il Vaticanus Lat. 362, non è una copia diretta dell'Escurialensis a II 10, e il Vaticanus Reginensis Lat. 589, nonostante la quasi perfetta concordanza con l'Escurialensis a II 10, non è una sua diretta copia ${ }^{22}$.

Il Remensis 1402 è molto simile al Bernensis Lat. 199, ma non è una sua diretta copia ${ }^{23}$. Il Remensis 1402 può trovarsi lievemente sotto l'influsso di quelle tradizioni manoscritte, che non influirono sui restanti manoscritti del gruppo "J", e i codici Bernensis Lat. 199, Remensis 1402, Vaticanus Reginensis Lat. 589, Andegavensis 154, Escurialensis a II 10, Vaticanus Lat. 362 e il Parisinus Lat. 16736 sono rappresentanti delle diverse file dei codici che derivano dal primo antenato del gruppo "J"; il Vaticanus Reginensis Lat. 589, invece, è un punto d'unione tra il Bernensis Lat. 199 e il Remensis 1402, da una parte, e dall'altra degli altri manoscritti ${ }^{24}$.

3. La famiglia " $Q$ "25. Ai quattro manoscritti che Cherf annoverò al gruppo "Q"26, aggiungemmo ben quattordici codici ${ }^{27}$. Questa famiglia si divide in due sottogruppi ${ }^{28}$.

Il Vaticanus Lat. 6075 concorda specialmente con il Vaticanus Lat. 1194 e il Vaticanus Lat. 5772 e appartiene allo stesso sottogruppo "Q". Ciò nonostante, non deriva direttamente né dal Vaticanus Lat. 1194, né dal Vaticanus Lat. $5772^{29}$.

${ }^{21}$ Cf. ibidem, p. 180.

${ }^{22}$ Cf. ibidem, p. 181.

${ }^{23}$ Cf. ibidem, p. 182-183.

${ }^{24}$ Cf. ibidem, p. 183.

${ }^{25}$ A proposito di questa famiglia, cf. idem, La famiglia " $Q$ ” della tradizione manoscritta della "Vita S. Pauli Primi Eremitae" di San Girolamo, DPa 5 (1992) 26-45; idem, La tecnica di preparazione di un'edizione critica. Un caso esemplativo: la familia "Q» della tradizione manoscritta della "Vita S. Pauli Primi Eremitae" di san Girolamo, in: Sanctitatis Causae. Motivi di santità e Cause di canonizzazione di alcuni maestri medioevali. In ricordo di Padre Louis-Jacques Bataillon, O.P., ed. M.M. Rossi - T. Rossi, Studia Pontificiae Universitatis a S. Thoma Aquinate in Urbe. Studi 2008. Nuova serie 12, Roma 2009, 11-43; B. Degórski, Kodeks Basilicanus A. 6 (alias E) a rodzina " Q" rękopiśmiennego przekazu "Vita Sancti Pauli Primi Eremitae” św. Hieronima, VoxP 31 (2011) t. 56, 485-493.

${ }^{26}$ Cf. Cherf, The Latin Manuscript Tradition, p. 115-117.

${ }^{27}$ Cf. Degórski, Przekaz łacińskich rękopisów, p. 187-204.

${ }^{28}$ Cf. ibidem, p. 205-207.

${ }^{29}$ Cf. ibidem, p. 208-209. 
Il Vaticanus Rossianus Lat. 287 e il Vaticanus Urbinas Lat. 48 sono più strettamente uniti con il Vaticanus Lat. 1201 che con il Vaticanus Lat. 6933 ${ }^{30}$. Tuttavia, il Vaticanus Rossianus Lat. 287 e il Vaticanus Urbinas Lat. 48 non furono copiati direttamente dal Vaticanus Lat. 1201; doveva esserci, tra di essi, un codice intermedio (o persino molteplici codici). Il Vaticanus Urbinas Lat. 48, però, non è una copia diretta del Vaticanus Rossianus Lat. 287. Ambo i manoscritti attinsero probabilmente da uno stesso codice ${ }^{31}$.

Il Vaticanus Barberinianus Lat. 621 e il Vaticanus Urbinas Lat. 51 hanno molte varianti in comune. Non provengono, comunque, direttamente dal $\mathrm{Va}$ ticanus Lat. 6933 o dal Vaticanus Lat. 1201. Derivano da un manoscritto che non si è conservato e che aveva una fonte comune con il Vaticanus Lat. 1201 e il Vaticanus Lat. $6933^{32}$. Le piccole differenze tra il Vaticanus Barberinianus Lat. 621 e il Vaticanus Urbinas Lat. 51 dimostrano che l'uno non è la diretta copia dell'altro ${ }^{33}$.

Il Casanatensis Codex 1898, il Vallicellanus Codex C. 55 e i codici Vaticanus Lat. 1199 e il Vaticanus Lat. 1200 formano un gruppo più compatto ${ }^{34}$.

Il Vallicellanus Codex C. 55, il Casanatensis Codex 1898 e il Vaticanus Lat. 1200 si avvicinano al Vaticanus Barberinianus Lat. 621 e al Vaticanus Urbinas Lat. 51. L'antenato del Vallicellanus Codex C. 55, del Casanatensis Codex 1898, del Vaticanus Lat. 1199 e del Vaticanus Lat. 1200, da una parte e, dall'altra parte, l'antenato del Vaticanus Barberinianus Lat. 621 e del Vaticanus Urbinas Lat. 51 derivano da un codice comune ${ }^{35}$.

Il Vaticanus Lat. 1199 ha delle aggiunte o delle lacune che non si incontrano nel Vallicellanus Codex C. 55, nel Casanatensis Codex 1898 e nel Vaticanus Lat. 1200. Probabilmente, perciò, il copista del Vaticanus Lat. 1199, servendosi di un codice comune al Vallicellanus Codex C. 55, al Casanatensis Codex 1898 e al Vaticanus Lat. 1200, si servì anche di un codice appartenente ad un'altra famiglia e introduceva, a volte, la lezione ivi contenuta, insieme con la lezione comune presente nel Vallicellanus Codex C. 55, nel Casanatensis Codex 1898 e nel Vaticanus Lat. $1200^{36}$.

Il Vaticanus Barberinianus Lat. 605 e il Vaticanus Urbinas Lat. 389 hanno varianti comuni. Cià nonostante, non provengono direttamente né dal Vaticanus Lat. 6933, né dal Vaticanus Lat. 1201, bensì da un terzo manoscritto che non si è conservato e che aveva una fonte comune con il Vaticanus Lat. 1201 e il Vaticanus Lat. 6933. Tuttavia, il Vaticanus Barberinianus Lat. 605 e il

\footnotetext{
${ }^{30}$ Cf. ibidem, p. 209-211.

${ }^{31}$ Cf. ibidem, p. 211-212.

${ }^{32}$ Cf. ibidem, p. 212-217.

${ }^{33}$ Cf. ibidem, p. 217.

${ }^{34}$ Cf. ibidem, p. 218-219.

${ }^{35}$ Cf. ibidem, p. 218-224.

${ }^{36}$ Cf. ibidem, p. 224.
} 
Vaticanus Urbinas Lat. 389 non furono copiati da uno stesso codice, perché differiscono lievemente tra di loro ${ }^{37}$.

Il Vaticanus Palatinus Lat. 179 concorda più strettamente con il Vaticanus Lat. 1201 e il Vaticanus Lat. 6933. Non proviene, però, direttamente né dal Vaticanus Lat. 1201, né dal Vaticanus Lat. 6933, perché a volte segue la lezione del Vaticanus Lat. 1201 e altre quella del Vaticanus Lat. 6933. Quindi, esso proviene da un terzo manoscritto che aveva una fonte comune con gli antenati del Vaticanus Lat. 1201 e del Vaticanus Lat. $6933^{38}$.

Il Vaticanus Palatinus Lat. 179, anche se non è strettamente unito con alcun codice (e ciò nonostante appartiene al gruppo "Q"), è vicino al Vaticanus Lat. 1201 e a quei codici che derivano da esso: al Vaticanus Rossianus Lat. 287 e, specialmente, al Vaticanus Urbinas Lat. 48. Quindi, l'antenato del Vaticanus Palatinus Lat. 179 e il codice da lui derivante, ossia il Vaticanus Lat. 1201 (la fonte del Vaticanus Rossianus Lat. 287 e del Vaticanus Urbinas Lat. 48), si incrociarono ${ }^{39}$.

Il Vallicellanus Tomus VI e il Casanatensis Codex 588 non sono uniti più strettamente con alcun codice del gruppo "Q". Ciò nonostante, si avvicinano di più al Vaticanus Lat. 1201 e al Vaticanus Lat. 6933, che al Vaticanus Lat. 5772, al Vaticanus Lat. 1194 e al Vaticanus Lat. 6075. Non provengono, però, direttamente né dal Vaticanus Lat. 1201, né dal Vaticanus Lat. 6933 perché, a volte, seguono la lezione del Vaticanus Lat. 1201 e, a volte, quella del Vaticanus Lat. 6933. Quindi, derivano da un codice che aveva con il Vaticanus Lat. 1201 e il Vaticanus Lat. 6933 una fonte comune dalla quale provengono anche: il Vaticanus Palatinus Lat. 179, il Vaticanus Barberinianus Lat. 621, il Vaticanus Urbinas Lat. 51, il Vaticanus Barberinianus Lat. 605, il Vaticanus Urbinas Lat. 389, il Casanatensis Codex 1898, il Vallicellanus Codex C. 55 e il Vaticanus Lat. 1200 e il Vaticanus Lat. 1199, perché tra di essi e il Vallicellanus Tomus VI e il Casanatensis Codex 588 intercorre una particolare vicinanza. Ciò nonostante, questi manoscritti, anche se appartengono al gruppo "Q", non sono strettamente uniti con alcun codice di questo gruppo. Essi, però, hanno una fonte comune con il Vaticanus Palatinus Lat. 179, il Vaticanus Barberinianus Lat. 621, il Vaticanus Urbinas Lat. 51, il Vaticanus Barberinianus Lat. 605, il Vaticanus Urbinas Lat. 389, il Casanatensis Codex 1898, il Vallicellanus Codex C. 55, il Vaticanus Lat. 1200 e il Vaticanus Lat. 1199º.

4. La famiglia "S"41. Alla famiglia "S", da noi creata e sconosciuta a Cherf e che proviene da Nonantola, annoverammo i codici: Sessorianus 41 (IX secolo), Vallicellanus Codex D. 2 (XIV secolo), Vaticanus Palatinus Lat. 853 (XIV

\footnotetext{
${ }^{37}$ Cf. ibidem, p. 225-231.

${ }^{38}$ Cf. ibidem, p. 230-231.

${ }^{39}$ Cf. ibidem, p. 231-235.

${ }^{40}$ Cf. ibidem, p. 235-237.

${ }^{41}$ A proposito di questa famiglia cf. idem, La famiglia " $S$ " della tradizione manoscritta della "Vita Sancti Pauli Primi Eremitae" di San Girolamo, DPa 6 (1993) 16-41.
} 
o XV secolo), Vaticanus Palatinus Lat. 844 (XV secolo), Vaticanus Chisianus Lat. H IV 119 (del 1460) ${ }^{42}$.

Il Sessorianus 41, il Vallicellanus Codex D. 2, il Vaticanus Palatinus Lat. 853, il Vaticanus Palatinus Lat. 844 formano un'unità più stretta, un sottogruppo. In esso, però, il Sessorianus 41 e il Vallicellanus Codex D. 2 sono più strettamente uniti. Lo stesso si verifica per il Vaticanus Palatinus Lat. 853 e il Vaticanus Lat. $844^{43}$.

Il Vaticanus Palatinus Lat. 844 si avvicina allo Scaphusianus 104 o, più precisamente parlando, ai codici che provengono dallo stesso gruppo dello Scaphusianus e che, però, non si sono conservati fino a noi. Infatti, il Vaticanus Palatinus Lat. 844 condivide con lo Scaphusianus molte varianti che non si incontrano negli altri manoscritti. Gli antenati, perciò, del Vaticanus Palatinus Lat. 844 e i manoscritti che derivano dallo Scaphusianus furono collegati tra di loro ${ }^{44}$.

Il Vallicellanus Codex D. 2, il Vaticanus Palatinus Lat. 853, il Vaticanus Lat. 844 e il Vaticanus Chisianus Lat. H IV 119 derivano da diverse linee, il che può essere visto da alcune lezioni comuni che essi condividono con le altre famiglie ${ }^{45}$.

5. La famiglia "W". Il Cherf annoverò al gruppo "W" sei manoscritti". Le nostre ricerche, però, introdussero qui delle ampie aggiunte ed emendamenti che riguardano i titoli dei capitoli e delle glosse marginali ${ }^{47}$. Inoltre, ai manoscriti elencati dal Cherf aggiungemmo altri quattro codici ${ }^{48}$.

Esiste una stretta parentela tra il Canonicianus Misc. 167 e il Novariensis LXI, l'Ambrosianus D 525 inf., l'Ambrosianus I 16 inf., il Vallicellanus Tomus XII, il Vaticanus Lat. 374, il Basilicanus B. 50 e il Vallicellanus Tomus $\mathrm{II}^{49}$. Ciò nonostante, una più stretta parentela intercorre tra il Novariensis LXI, 1'Ambrosianus D 525 inf., l'Ambrosianus I 61 inf., il Vallicellanus Tomus XII, il Vaticanus Lat. 374, il Basilicanus B. 50 e il Vallicellanus Tomus II $^{50}$. Dentro questo gruppo, il Vallicellanus Tomus XII e il Tomus II sono più strettamente collegati. Essi comunque non provengono direttamente né dall'Ambrosianus D 525 inf., né dal Canonicianus Misc. 167. Dal momento, però, che il Vallicellanus Tomus XII e il Tomus II sono uniti con l'Ambrosianus D 525 inf. e con il Canonicianus Misc. 167, bisogna dire che il loro antenato aveva una fonte comune con l'Ambrosianus D 525 inf. e con il Canonicianus

\footnotetext{
${ }^{42}$ Cf. idem, Przekaz łacińskich rękopisów, p. 240-245.

${ }^{43}$ Cf. ibidem, p. 246-250.

${ }^{44}$ Cf. ibidem, p. 251-252.

${ }^{45}$ Cf. ibidem, p. 253-276.

${ }^{46}$ Cf. Cherf, The Latin Manuscript Tradition, p. 118-119.

${ }^{47}$ Cf. Degórski, Przekaz łacińskich rękopisów, p. 279-290.

${ }^{48}$ Cf. ibidem, p. 286-290.

${ }^{49}$ Cf. ibidem, p. 291.

${ }^{50}$ Cf. ibidem, p. 291-292.
} 
Misc. 167. Il Vallicellanus Tomus XII e il Tomus II vanno considerati gemelli e non copie ${ }^{51}$.

Il Basilicanus B. 50 non deriva dallo stesso manoscritto da cui dipendono l'Ambrosianus D 525 inf. e il Canonicianus Misc. 167, bensì da un manoscritto che aveva con essi una fonte comune ${ }^{52}$.

Il Vaticanus Lat. 374 subì l'influsso delle altre famiglie (specialmente della "D" e della "Q") $)^{53}$.

Il Novariensis LXI e l'Ambrosianus I 61 inf. hanno in comune otto varianti; sono gemelli, non copie ${ }^{54}$.

6. La famiglia "Z". Il Cherf annoverò alla famiglia "Z" (originaria della valle della Loira) cinque codici ${ }^{55}$, e noi aggiungemmo ad essi il Parisinus Lat. $10840^{56}$.

Il Sangallensis 552, il Remensis 428, il Bruxellensis 8344/46 e il Parisinus Lat. 10840 sono più strettamente uniti rispetto ai restanti manoscritti di questo gruppo: il Catalaunensis 53, il Laurentianus-Ashburnhamensis 58 e il Parisinus Lat. 10840 formano un sottogruppo che è più vicino al Sangallensis 552 e al Remensis 428 che al Bruxellensis 8344/46. Inoltre, il Sangallensis 552 e il Remensis 428 avevano una fonte comune ${ }^{57}$.

\section{LA VITA S. PAULI PRIMI EREMITAE NELLE PIÙ ANTICHE EDIZIONI A STAMPA}

1. La Vita S. Pauli Primi Eremitae stampata a Venezia nel 1511. Presentiamo il più antico, come pare, testo stampato della Vita S. Pauli nel 1511 a Venenzia, a cura del libraio Mattia Milcher di Buda ${ }^{58}$.

a) La divisione del testo. Dopo il titolo: "Uita sancti pauli primi heremite",59, segue un testo dell'editore che è assente in san Girolamo:

"Incipit vita Sancti Pauli primi heremite, a beato Hieronymo conscripta et a sancta Romana Ecclesia cum omni honore recepta, vt habetur in decretis. xv.d.c.Sancta romana $. \S . i t e m$ vitas, ab omni vitio scriptorum, non ex proprio

${ }^{51}$ Cf. ibidem, p. 292-293.

${ }^{52}$ Cf. ibidem, p. 294-295.

${ }^{53}$ Cf. ibidem, p. 295.

${ }^{54} \mathrm{Cf}$. ibidem.

${ }^{55}$ Cf. Cherf, The Latin Manuscript Tradition, p. 121-122.

${ }^{56}$ Cf. Degórski, Przekaz tacińskich rękopisów, p. 304.

${ }^{57}$ Cf. ibidem, p. 307-309.

${ }^{58}$ Cf. Vita diui Pauli primi heremite. Matthias Milcher librarius Budensis [...] Venetiis anno virginei partus 1511, die primo Decembris. Impensis Matthie Milcher librarii Budensis, arte autem Jacobi Pentii de Leucho, Reprint der Originalausgabe von 1511 mit einer Einfürung von S. Świdziński, Archivum Ordinis Sancti Pauli Primi Eremitae II: Fontes, vol. 10, ed. S. Świdziński, Coesfeld 2009.

${ }^{59}$ Cf. Vita diui Pauli 1. 
sensu, sed ex correctissimis exemplaribus, per plures librarias requisitis, cum omni diligentia exactissime emendata" ${ }^{\circ}$.

Dopo questo testo, segue un'introduzione dell'editore al prologo geronimiano:

"Et primo prologus eiusdem beati hieronymi presbyteri, in quo ostenditur quis fuerit primus heremita"

Segue il prologo: "Inter multos" 62 che termina con le parole: "compertum" ${ }^{63}$ habetur" ${ }^{64}$. Nel prologo troviamo un'ulteriore suddivisione indicata con un segno simile alla lettera "C". Il prologo è diviso nel modo seguente: 1) dalle parole "Inter multos" ${ }^{65}$, alle parole: "sententia videtur" ${ }^{66} ; 2$ ) dalle parole: "Igitur quia de Antonio"67 fino alla fine del prologo: "compertum ${ }^{68}$ habetur" ${ }^{69}$.

Dopo il prologo segue: "Incipit vita sancti Pauli primi heremite. Caput I.". Il cap. I abbraccia il testo dalle parole: "Sub decio et valeriano" 70 alle parole: "exempla subicimus" fide" 72 e finisce con le parole: "mihi promisit, ostendet" 73 . Il cap. II, che secondo la divisione contenuta nella PL si estente fino al cap. VII, è qui diviso nel seguente modo: 1) dall'inizio: "Perseuerantem in fide" "74 alle parole: "magnitudo praeripuit ${ }^{75}$ "76 (ciò corrisponde al cap. III nella PL); 2) dalle parole: "Per idem ergo tempus" 77 alle parole: "iunctus Antonius est" PL); 3) dalle parole: "Igitur adamato"79 fino alla fine del capitolo: "promisit, ostendet" $" 80$ (il cap. VI e una parte del cap. VII nella PL). Il cap. III si estende

\footnotetext{
${ }^{60} \mathrm{Cf}$. ibidem.

${ }^{61} \mathrm{Cf}$. ibidem.

${ }^{62}$ Cf. Hieronymus, Vita Sancti Pauli 1, ed. Degórski, p. 66; Vita diui Pauli 1.

${ }^{63}$ Certo: "cōpertū".

${ }^{64}$ Cf. Hieronymus, Vita Sancti Pauli 1, ed. Degórski, p. 72-73; Vita diui Pauli 1.

${ }^{65}$ Cf. Hieronymus, Vita Sancti Pauli 1, ed. Degórski, p. 66; Vita diui Pauli 1.

${ }^{66}$ Cf. Hieronymus, Vita Sancti Pauli 1, ed. Degórski, p. 71; Vita diui Pauli 1.

${ }^{67}$ Cf. Hieronymus, Vita Sancti Pauli 1, ed. Degórski, p. 71; Vita diui Pauli 1.

${ }^{68}$ Certo: "cōpertū".

${ }^{69}$ Cf. Hieronymus, Vita Sancti Pauli 1, ed. Degórski, p. 73; Vita diui Pauli 1.

${ }^{70}$ Cf. Hieronymus, Vita Sancti Pauli 2, ed. Degórski, p. 74; Vita diui Pauli 1.

${ }^{71}$ Cf. Hieronymus, Vita Sancti Pauli 2, ed. Degórski, p. 76; Vita diui Pauli 2.

${ }^{72}$ Cf. Hieronymus, Vita Sancti Pauli 3, ed. Degórski, p. 76; Vita diui Pauli 2.

${ }^{73}$ Cf. Hieronymus, Vita Sancti Pauli 7, ed. Degórski, p. 100; Vita diui Pauli 3.

${ }^{74}$ Cf. Hieronymus, Vita Sancti Pauli 3, ed. Degórski, p. 76; Vita diui Pauli 2.

${ }^{75}$ Certo: "pripuit". "-" super "p" scriptum est.

${ }^{76}$ Cf. Hieronymus, Vita Sancti Pauli 3, ed. Degórski, p. 81-82; Vita diui Pauli 2.

${ }^{77}$ Cf. Hieronymus, Vita Sancti Pauli 4, ed. Degórski, p. 82; Vita diui Pauli 2.

${ }^{78}$ Cf. Hieronymus, Vita Sancti Pauli 5, ed. Degórski, p. 92; Vita diui Pauli 3.

${ }^{79}$ Cf. Hieronymus, Vita Sancti Pauli 6, ed. Degórski, p. 92-93; Vita diui Pauli 3.

${ }^{80}$ Cf. Hieronymus, Vita Sancti Pauli 7, ed. Degórski, p. 100; Vita diui Pauli 3.
} 
dalle parole: "Nec plura his" $" 11$ alle parole: "incertum habemus" (= la parte finale del cap. VII nella PL). Il cap. IV dalle parole: "Stupens itaque" role: "non posse confideret" 84 . Questo corrisponde al cap. VIII e all'inizio del cap. IX della PL. Il capitolo è diviso: 1) dall' inizio: "Stupens itaque" ${ }^{85}$ fino alle parole: "animal aufugit" 86 (ciò corrisponde quasi a tutto il cap. VIII della PL); 2) dalle parole: "Hoc ne cuiquam" 87 , alle parole: "allatum est" 88 (= la parte finale del cap. VIII nella PL); 3) dalle parole: "Sed ut propositum" $"$, alle parole: "non posse confideret" $"{ }^{\circ}$ (= l'inizio del cap. IX nella PL). Il cap. V dalle parole: "Per nox secundas"91, alle parole: "in communi referuntur"92. Ciò corrisponde quasi del tutto al cap. IX della PL. Il cap. V non è diviso. Il cap. VI dalle parole: "Et post secundum"93, alle parole: "spiritum redderet"94. Ciò corrisponde quasi a tutti i capp. X-XII e all'inizio del cap. XIII della PL. Il capitolo è diviso nel seguente modo: 1) dalle parole: "Et post secundum"95, alle parole: "mortis alleuiaretur"96; 2) dalle parole: "Stupefactus Antonius" "97, fino alla fine del capitolo: "spiritum redderet" ". Il cap. VII si estende dalle parole: "Cum iam dies" $"$, alle parole: "gestus precaretur" ${ }^{100}$. Ciò corrisponde alla seconda parte del cap. XIV e di tutto il cap. XV della PL. Il cap. VII non è diviso. Il cap. VIII si estende dalle parole: "Igitur obuoluto"101 sino alla fine: "cum regnis suis"102. Questo corrisponde ai capp. XVI-XVIII nella PL. Il capitolo è stato diviso nel modo seguente: 1) dalle parole: "Igitur obuoluto"103, alle parole: "ut abirent imperauit"104 (ciò corrisponde alla prima metà del cap. XVI nella PL); 2) dalle

\footnotetext{
${ }^{81}$ Cf. Hieronymus, Vita Sancti Pauli 7, ed. Degórski, p. 100-101; Vita diui Pauli 3.

${ }^{82}$ Cf. Hieronymus, Vita Sancti Pauli 7, ed. Degórski, p. 105; Vita diui Pauli 4.

${ }^{83}$ Cf. Hieronymus, Vita Sancti Pauli 8, ed. Degórski, p. 105; Vita diui Pauli 4.

${ }^{84}$ Cf. Hieronymus, Vita Sancti Pauli 9, ed. Degórski, p. 118; Vita diui Pauli 4.

${ }^{85}$ Cf. Hieronymus, Vita Sancti Pauli 8, ed. Degórski, p. 105; Vita diui Pauli 4.

${ }^{86}$ Cf. Hieronymus, Vita Sancti Pauli 8, ed. Degórski, p. 114; Vita diui Pauli 4.

${ }^{87}$ Cf. Hieronymus, Vita Sancti Pauli 8, ed. Degórski, p. 114; Vita diui Pauli 4.

${ }^{88}$ Cf. Hieronymus, Vita Sancti Pauli 8, ed. Degórski, p. 116-117; Vita diui Pauli 4.

${ }^{89}$ Cf. Hieronymus, Vita Sancti Pauli 9, ed. Degórski, p. 117; Vita diui Pauli 4.

${ }^{90}$ Cf. Hieronymus, Vita Sancti Pauli 9, ed. Degórski, p. 118; Vita diui Pauli 4.

${ }^{91}$ Cf. Hieronymus, Vita Sancti Pauli 9, ed. Degórski, p. 119; Vita diui Pauli 5.

${ }^{92}$ Cf. Hieronymus, Vita Sancti Pauli 9, ed. Degórski, p. 129; Vita diui Pauli 5.

${ }^{93}$ Cf. Hieronymus, Vita Sancti Pauli 10, ed. Degórski, p. 130; Vita diui Pauli 5.

${ }^{94}$ Cf. Hieronymus, Vita Sancti Pauli 14, ed. Degórski, p. 153; Vita diui Pauli 7.

${ }^{95}$ Cf. Hieronymus, Vita Sancti Pauli 10, ed. Degórski, p. 130; Vita diui Pauli 5.

${ }^{96}$ Cf. Hieronymus, Vita Sancti Pauli 12, ed. Degórski, p. 145-146; Vita diui Pauli 6.

${ }^{97}$ Cf. Hieronymus, Vita Sancti Pauli 12, ed. Degórski, p. 146; Vita diui Pauli 6.

${ }^{98}$ Cf. Hieronymus, Vita Sancti Pauli 14, ed. Degórski, p. 153; Vita diui Pauli 7.

${ }^{99}$ Cf. Hieronymus, Vita Sancti Pauli 14, ed. Degórski, p. 153-154; Vita diui Pauli 7.

${ }^{100}$ Cf. Hieronymus, Vita Sancti Pauli 15, ed. Degórski, p. 158; Vita diui Pauli 7.

${ }^{101}$ Cf. Hieronymus, Vita Sancti Pauli 16, ed. Degórski, p. 159; Vita diui Pauli 7.

${ }^{102}$ Cf. Hieronymus, Vita Sancti Pauli 18, ed. Degórski, p. 179-180; Vita diui Pauli 7.

${ }^{103}$ Cf. Hieronymus, Vita Sancti Pauli 16, ed. Degórski, p. 159; Vita diui Pauli 7.

${ }^{104}$ Cf. Hieronymus, Vita Sancti Pauli 16, ed. Degórski, p. 168; Vita diui Pauli 8.
} 
parole: "curuauit humeros"105, alle parole: "vestitus est"106 (ciò corrisponde al resto del cap. XVI della PL); 3) dalle parole: "Libet in fine" 107 sino alla fine: "cum regnis suis"108. Ciò corrisponde ai cap. XVII e XVIII della PL.

Dopo la Vita $S$. Pauli di san Girolamo troviamo la seguende chiusa dell'editore:

"Finit vita Sancti Pauli primi heremite. Maximo labore limatum, castigatum, politumque opus, rasor aut pictor corumpere noli, quod si in scedis tuis aliquid superfuit vel deficit ne mireris. Hec pagella non tibi deest nisi tu ei desis"109.

b) Un tentativo di classificazione. La Vita diui Pauli condivide con la famiglia "A" (bavarese) un'unica lezione: anziché "animas cupiebat iugulare"110, presenta: "animas quaerebat ${ }^{111}$ vigilare"112.

Con il gruppo "R" condivide la seguente lezione: anziché "lutulenta aqua uiuit"113, legge: "lutulentam aquam bibebat"114. A dir il vero, la famigia " $R$ " ha: "lutulentam aquam bibens".

Con la famiglia " $Z$ " condivide la seguente variante: anziché "a scelere reuocauerunt" 115 , presenta: "a scelere reuocabat" ${ }^{116}$. La Vita diui Pauli si avvicina anche al gruppo " $Z$ " nel seguente punto: mentre san Girolamo scrive: "Referebat postea"117, la famiglia " $Z$ " aggiunge: "Referebat quoque postea" 118 , e la Vita diui Pauli ha la versione: "Referebatque postea"119.

Ciò nonostante, non possiamo classificare la Vita diui Pauli in alcuna di queste famiglie. Infatti, il suo testo è il frutto delle posteriori contaminazioni subite dalla Vita S. Pauli.

2. La Vita S. Pauli Primi Eremitae stampata a Cracovia nel 1532. Presentiamo qui il testo stampato della Vita Pauli, contenuto nel Decalogus de beato Paulo primo ed edito nel 1532 a Cracovia presso la tipografia di Floriano Ungler ${ }^{120}$.

\footnotetext{
${ }^{105}$ Cf. Hieronymus, Vita Sancti Pauli 16, ed. Degórski, p. 168; Vita diui Pauli 8.

${ }^{106}$ Cf. Hieronymus, Vita Sancti Pauli 16, ed. Degórski, p. 171; Vita diui Pauli 8.

${ }^{107}$ Cf. Hieronymus, Vita Sancti Pauli 17, ed. Degórski, p. 172; Vita diui Pauli 8.

${ }^{108}$ Cf. Hieronymus, Vita Sancti Pauli 18, ed. Degórski, p. 179-180; Vita diui Pauli 8.

${ }^{109}$ Cf. Vita diui Pauli 8.

${ }^{110}$ Cf. Hieronymus, Vita Sancti Pauli 2, ed. Degórski, p. 75.

${ }^{111}$ Certo: "qrebat". "-" super "q" scriptum est.

${ }^{112}$ Cf. Vita diui Pauli 1.

${ }^{113}$ Cf. Hieronymus, Vita Sancti Pauli 6, ed. Degórski, p. 94.

${ }^{114} \mathrm{Cf}$. Vita diui Pauli 3.

${ }^{115}$ Cf. Hieronymus, Vita Sancti Pauli 4, ed. Degórski, p. 86.

${ }^{116} \mathrm{Cf}$. Vita diui Pauli 2.

${ }^{117}$ Cf. Hieronymus, Vita Sancti Pauli 15, ed. Degórski, p. 156.

${ }^{118}$ Cf. Degórski, Przekaz łacińskich rękopisów, p. 306.

${ }^{119}$ Cf. Vita diui Pauli 7.

${ }^{120} \mathrm{Cf}$. Decalogus de beato Paulo primo heremita comportatus per reverendum patrem fratrem Gregorium de Gyengyes protunc priorem sancti Stephani Rotundi in Vrbe cum annotationibus in
} 
a) La divisione del testo. Il testo della Vita Pauli contenuto nel Decalogus è diviso nel seguente modo: 1) Il prologo. Dalle parole: "Prologus Beati Iheronymi presbyteri, in vitam sancti Pauli primi heremite. Inter multos" ${ }^{121}$, alle: "compertum habetur" 122 . 2) In seguito: 1'"incipit": "Incipit vita sancti Pauli primi heremite"123. 3) Dopo 1' "incipit", la Vita Pauli inizia con le parole: "Sub Decio et Valeriano" "124. 4) Il testo della Vita Pauli prosegue sino alla fine (senza alcuna divisione in capitoli), ovverosia sino alle parole: "cum penis suis" 125. Al testo fu aggiunta la parola: "Finis"126.

Anche se il testo della Vita Pauli non è diviso in capitoli, fu articolato in paragrafi tramite il segno "q". Tale divisione si presenta nel modo seguente: 1) Il I par.: dalle parole "Cuius crudelitas"127, alle parole: "sartagines superasset"128. 2) Il II: dalle parole: "Alium iuuenili"129, alle parole: "magnitudo superauit"130.3) Il III: dalle parole: "Per idem ergo"131, alle parole "genuerat transorbebat"132.4) Il IV: dalle parole: "Erant preterea"133, alle parole: "iunctus est Anthonius"134. 5) Il V: dalle parole: "Adamato igitur"135, alle parole "vnde digressus sum"136. 6) Il VI: dalle parole: "Cum iam centum tredecim"137, alle parole "deberet properare"138. 7) Il VII: dalle parole: "Illico erumpente"139, alle parole "mirantis euanuit" 140 . 8) L'VIII paragrafo non è preceduto dal segno "ब", ma comincia a linea e le sue prime parole sono scritte con caratteri più grandi. Con caratteri più grandi sono anche scritte le parole che iniziano il

margine adiecti, Cracouie per Florianum Unglerum 1532, Archivum Ordinis Sancti Pauli Primi Eremitae II: Fontes, vol. 9, ed. S. Świdziński, Coesfeld 2008, 8-18. Cf. anche Anhang I [edizione critica e traduzione polacca della Vita S. Pauli Primi Eremitae di san Girolamo, contenuta in G. Gyengyes, Decalogus de beato Paulo primo heremita, Cracoviae 1532], ed. B. Degórski, in: Archivum Ordinis Sancti Pauli Primi Eremitae II: Fontes, vol. 9, ed. S. Świdziński, Coesfeld 2008, 170-187.

${ }^{121}$ Cf. Decalogus de beato Paulo 8; Hieronymus, Vita Sancti Pauli 1, ed. Degórski, p. 66-67.

${ }^{122}$ Cf. Decalogus de beato Paulo 8; Hieronymus, Vita Sancti Pauli 1, ed. Degórski, p. 72-73.

${ }^{123}$ Cf. Decalogus de beato Paulo 9.

${ }^{124}$ Cf. ibidem; Hieronymus, Vita Sancti Pauli 2, ed. Degórski, p. 74.

${ }^{125}$ Cf. Decalogus de beato Paulo 18; Hieronymus, Vita Sancti Pauli 18, ed. Degórski, p. 178-180.

${ }^{126} \mathrm{Cf}$. Decalogus de beato Paulo 18.

${ }^{127}$ Cf. ibidem 9; Hieronymus, Vita Sancti Pauli 2, ed. Degórski, p. 76.

${ }^{128}$ Cf. Decalogus de beato Paulo 9; Hieronymus, Vita Sancti Pauli 3, ed. Degórski, p. 77.

${ }^{129}$ Cf. Decalogus de beato Paulo 9; Hieronymus, Vita Sancti Pauli 3, ed. Degórski, p. 77.

${ }^{130}$ Cf. Decalogus de beato Paulo 10; Hieronymus, Vita Sancti Pauli 3, ed. Degórski, p. 81-82.

${ }^{131}$ Cf. Decalogus de beato Paulo 10; Hieronymus, Vita Sancti Pauli 4, ed. Degórski, p. 82.

${ }^{132}$ Cf. Decalogus de beato Paulo 10; Hieronymus, Vita Sancti Pauli 5, ed. Degórski, p. 89-90.

${ }^{133}$ Cf. Decalogus de beato Paulo 10; Hieronymus, Vita Sancti Pauli 5, ed. Degórski, p. 90.

${ }^{134}$ Cf. Decalogus de beato Paulo 11; Hieronymus, Vita Sancti Pauli 5, ed. Degórski, p. 92.

${ }^{135}$ Cf. Decalogus de beato Paulo 11; Hieronymus, Vita Sancti Pauli 6, ed. Degórski, p. 92-93.

${ }^{136}$ Cf. Decalogus de beato Paulo 11; Hieronymus, Vita Sancti Pauli 7, ed. Degórski, p. 96.

${ }^{137}$ Cf. Decalogus de beato Paulo 11; Hieronymus, Vita Sancti Pauli 7, ed. Degórski, p. 97.

${ }^{138}$ Cf. Decalogus de beato Paulo 11; Hieronymus, Vita Sancti Pauli 7, ed. Degórski, p. 99.

${ }^{139}$ Cf. Decalogus de beato Paulo 11; Hieronymus, Vita Sancti Pauli 7, ed. Degórski, p. 99.

${ }^{140}$ Cf. Decalogus de beato Paulo 12; Hieronymus, Vita Sancti Pauli 7, ed. Degórski, p. 104. 
par. VIII: "Verum hoc vtrum"141. Il par. VIII termina: "exiuit sonus eius"142. 9) Il IX inizia con le parole: "Talia eo loquente"143, e finisce: "propositum prosequar"144. 10) Il X comincia: "Anthonius cepta regione"145, e finisce: "sera, obfirmauit"146. 11) L'XI comincia con: "Tunc Anthonius"147, e termina con le parole: "Talia prestabat memorans"148. 12) Il XII comincia con: "Atque huic responsum"149, e termina con: "errore rapiantur?"150. 13) Il XIII comincia: "Inter has sermocinationes"151, e finisce: "duplicauit annonam"152. 14) Il XIV inizia con le parole: "Igitur domino"153, e termina con: "adhuc exemplo"154. 15) Il XV comincia: "Quamobrem perge"155, e termina: "quam cito recedis"156. 16) Il XVI comincia: "Referebatque preterea"157, e termina: "vestitus est"158. 17) Il XVII inizia con: "Libet in fine"159, e finisce con: "cum penis suis" ${ }^{160}$. Alla fine del testo geronimianu fu aggiunta la parola: "Finis"161. La divisione della Vita Pauli, contenuta nel Decalogus, corrisponde quasi esattamente a quella della Patrologia Latina ${ }^{162}$.

\section{b) Un tentativo di classificazione.}

1. Le aggiunte che si trovano anche negli altri manoscritti della Vita S. Pauli

1) Dopo la parola: "studia"163, il Decalogus aggiunge: $<$ Monachorum $>164$. Tale aggiunta è anche presente nei codici: Angelicus 234 ${ }^{165}$, Casanatensis

${ }^{141}$ Cf. Decalogus de beato Paulo 12; Hieronymus, Vita Sancti Pauli 7, ed. Degórski, p. 104.

${ }^{142}$ Cf. Decalogus de beato Paulo 12; Hieronymus, Vita Sancti Pauli 8, ed. Degórski, p. 111.

${ }^{143}$ Cf. Decalogus de beato Paulo 12; Hieronymus, Vita Sancti Pauli 8, ed. Degórski, p. 111.

${ }^{144}$ Cf. Decalogus de beato Paulo 13; Hieronymus, Vita Sancti Pauli 8-9, ed. Degórski, p. 116-117.

${ }^{145}$ Cf. Decalogus de beato Paulo 13; Hieronymus, Vita Sancti Pauli 9, ed. Degórski, p. 117.

${ }^{146}$ Cf. Decalogus de beato Paulo 13; Hieronymus, Vita Sancti Pauli 9, ed. Degórski, p. 123-124.

${ }^{147}$ Cf. Decalogus de beato Paulo 13; Hieronymus, Vita Sancti Pauli 9, ed. Degórski, p. 124.

${ }^{148}$ Cf. Decalogus de beato Paulo 14; Hieronymus, Vita Sancti Pauli 9, ed. Degórski, p. 127.

${ }^{149}$ Cf. Decalogus de beato Paulo 14; Hieronymus, Vita Sancti Pauli 9, ed. Degórski, p. 127.

${ }^{150}$ Cf. Decalogus de beato Paulo 14; Hieronymus, Vita Sancti Pauli 10, ed. Degórski, p. 132.

${ }^{151}$ Cf. Decalogus de beato Paulo 14; Hieronymus, Vita Sancti Pauli 10, ed. Degórski, p. 132.

${ }^{152}$ Cf. Decalogus de beato Paulo 14; Hieronymus, Vita Sancti Pauli 10, ed. Degórski, p. 134.

${ }^{153}$ Cf. Decalogus de beato Paulo 14; Hieronymus, Vita Sancti Pauli 11, ed. Degórski, p. 135.

${ }^{154}$ Cf. Decalogus de beato Paulo 15; Hieronymus, Vita Sancti Pauli 12, ed. Degórski, p. 143-144.

${ }^{155}$ Cf. Decalogus de beato Paulo 15; Hieronymus, Vita Sancti Pauli 12, ed. Degórski, p. 144.

${ }^{156}$ Cf. Decalogus de beato Paulo 16; Hieronymus, Vita Sancti Pauli 14, ed. Degórski, p. 155-156.

${ }^{157}$ Cf. Decalogus de beato Paulo 16; Hieronymus, Vita Sancti Pauli 15, ed. Degórski, p. 156.

${ }^{158}$ Cf. Decalogus de beato Paulo 18; Hieronymus, Vita Sancti Pauli 16, ed. Degórski, p. 171.

${ }^{159}$ Cf. Decalogus de beato Paulo 18; Hieronymus, Vita Sancti Pauli 17, ed. Degórski, p. 172.

${ }^{160}$ Cf. Decalogus de beato Paulo 18; Hieronymus, Vita Sancti Pauli 18, ed. Degórski, p. 179-180.

${ }^{161} \mathrm{Cf}$. Decalogus de beato Paulo 18.

${ }^{162}$ Cf. PL 23, 17-30.

${ }^{163}$ Cf. Hieronymus, Vita Sancti Pauli 1, ed. Degórski, p. 69.

${ }^{164} \mathrm{Cf}$. Decalogus de beato Paulo 8.

${ }^{165}$ L'aggiunta contenuta in questo codice è la seguente: " $<$ monacorum $>$ ". 
Codex 253 ${ }^{166}$, Iesuiticus 973, Vallicellanus Codex C. $74^{167}$, e nei codici: Vaticanus Lat. 349, 358, 363, 5087 ${ }^{168}$, 5119 ${ }^{169}, 5257$, Vaticanus Chisianus Lat. H IV $119^{170}$, e nei codici: Vaticanus Palatinus Lat. 179 i 860, nonché Iagellonicus Cracoviensis 2265 AA XII $9^{171}$.

La parola $<$ monachorum $>$ fu aggiunta a secunda manu super lineam anche nel secondo testo del codice Sanctae Mariae Maioris 1. Questi codici appartengono alla famiglia "I", eccezion fatta per i codici: Iagellonicus Cracoviensis 2265 AA XII 9 (non classificabile), Vaticanus Chisianus Lat. H IV 119 (appartenente alla famiglia "S"), Vaticanus Palatinus Lat. 179 (appartenente alla famiglia "Q"), Vaticanus Lat. 5257 (non classificabile).

2) Alle parole "calcaneo tenus hominem"172 il Decalogus aggiunge: "calcaneotenus hominem $<$ fuisse $>{ }^{173}$ ". L'aggiunta si trova anche nei codici: Cantabrigiensis $389^{174}$ (appartenente alla famiglia “D”), Casanatensis Codex 718 (non classificabile), Casanatensis Codex 1898 (appartenente alla famiglia "Q"), Lateranensis Codex A. 79 (non classificabile), Parisinus Lat. 11749 (appartenente alla famiglia "D"), Vallicellanus Codex C. 55 (appartenente alla famiglia "Q"), Vaticanus Barberinianus Lat. $469^{175}$ (non classificabile), Vaticanus Barberinianus Lat. 605 (appartenente alla famiglia "Q"), Vaticanus Ottobonianus Lat. 120 (appartenente alla famiglia "D"), i codici Vaticanus Lat. 1199 e 1200 (appartenenti alla famiglia “Q”), Wigorniensis F 48 (appartenente alla famiglia "L"). Inoltre, <fuisse> venne aggiunto da una seconda mano, super lineam, nel codice Cotton Caligula A XV (appartenente alla famiglia "K").

3) Alla parola "multa"176 il Decalogus aggiunge: "multa <alia>"177. L'aggiunta è presente anche nei codici: Basilicanus B. 50 (appartenente alla famiglia "W"), Berolinensis Manuscriptus Theologicus Lat. $7800^{178}$ (appartenente alla famiglia "E"), Bruxellensis 7882 (appartenente alla famiglia "E"), Bruxellensis 9398/99 (appartenente alla famiglia “E”), Casanatensis Codex 253 (appartenente alla famiglia "I"), Casanatensis Codex 718 (non classificabile),

\footnotetext{
${ }^{166}$ L'aggiunta contenuta in questo codice è la seguente: " $<$ monacorum $>$ ".

${ }^{167}$ L'aggiunta è la seguente: "<monaorum>"; 2 m. super lineam "ch" addidit.

${ }^{168}$ L'aggiunta contenuta in questo codice è la seguente: " $<$ monacorum omnium $>$ ".

${ }^{169}$ L'aggiunta contenuta in questo codice è la seguente: " $<$ monacorum $>$ ".

${ }^{170}$ L'aggiunta contenuta in questo codice è la seguente: " $<$ monacorum $>$ ".

${ }^{171}$ A proposito del codice cf. B. Degórski, Un tardivo manoscritto di Cracovia contenente la

"Vita Sancti Pauli Primi Eremitae" di San Girolamo, VoxP 8 (1988) t. 14, 429-434.

${ }^{172}$ Cf. Hieronymus, Vita Sancti Pauli 1, ed. Degórski, p. 70.

${ }^{173} \mathrm{Cf}$. Decalogus de beato Paulo 8.

${ }^{174}$ L'aggiunta contenuta in questo codice è la seguente: "subterreno specu crinitum calcaneo tenus $<$ fuisse $>$ hominem".

${ }^{175}$ Sub fuisse secunda manus, ut videtur, puncta scripsit.

${ }^{176} \mathrm{Cf}$. Hieronymus, Vita Sancti Pauli 1, ed. Degórski, p. 71.

${ }^{177} \mathrm{Cf}$. Decalogus de beato Paulo 8.

${ }^{178} \mathrm{La}$ parola "multa" non appare in questo manoscritto (non è visibile), perché il codice
} è qui guasto. 
Erlangensis 161 (appartenente alla famiglia “E”), Lateranensis Codex A. 79 (non classificabile), Monacensis 4597 (appartenente alla famiglia "D"), Monacensis $5157^{179}$ (appartenente alla famiglia “E”), Monacensis $17137^{180}$ (appartenente alla famiglia "E"), Monacensis 22033 (appartenente alla famiglia "D"), Parisinus Lat. 5314 (non classificabile), Parisinus Lat. 5342 (appartenente alla famiglia "R"), Remensis 1390 (K 769) (appartenente alla famiglia "E"), Vallicellanus Codex C. 74 (appartenente alla famiglia "I"), Vallicellanus Codex D. 2 (appartenente alla famiglia "S"), Vallicellanus Tomus III (non classificabile), e nei codici: Vaticanus Lat. 349, 358, 363, 5087, 5119 (appartenenti alla famiglia "I") 181,7014 (non classificabile), Vaticanus Barberinianus Lat. 469 (non classificabile), Vaticanus Chisianus Lat. A IV 90 (appartenente alla famiglia "I"), Vaticanus Chisianus Lat. H IV 119 (appartenente alla famiglia "S"), Vaticanus Palatinus Lat. 860 (appartenente alla famiglia "I"), Vindobonensis Sanctae Mariae ad Scotos 409 (non classificabile).

4) Alle parole "quo se conferret?"182 il Decalogus aggiunge: "quo se verteret $<$ nesciebat>" $>$ " L' L'aggiunta si trova anche nei codici: Berolinensis Manuscriptus Theologicus Lat. 780 (appartenente alla famiglia "E"), Bruxellensis 8344/46 (appartenente alla famiglia “Z”), Bruxellensis 9398/99 (appartenente alla famiglia "E"), Cantabrigiensis 389 (appartenente alla famiglia "D"), Cantabrigiensis Mm. 4. 28 (appartenente alla famiglia "E”), Erlangensis 161 (appartenente alla famiglia "E"), Farfensis 5 (270) (non classificabile), e nei codici: Monacensis 2540, 17137 (appartenenti alla famiglia "E"), Parisinus Lat. 5342, 16050 (appartenenti alla famiglia "R") ${ }^{184}$, Remensis 1390 (appartenente alla famiglia "E"), Vaticanus Ottobonianus Lat. 120 (appartenente alla famiglia “D”), Vindobonensis Lat. 420 (non classificabile), Vindobonensis Sanctae Mariae ad Scotos 409 (non classificabile), Wigorniensis F 48 (appartenente alla famiglia "L"). Tale aggiunta è presente anche nel testo della $P L^{185}$.

Nel Vaticanus Reginensis Lat. 589 (appartenente alla famiglia "J") l'aggiunta $<$ nesciebat $>$ si trova in margine, e nel Vaticanus Lat. 349 (appartenente alla famiglia "I") troviamo $<$ nesciuit $>$.

5) Alle parole "Qui sim, unde [...]"186 il Decalogus aggiunge: "Quis sim, $<$ et> vnde [...]"187. L'aggiunta <et> è presente anche nei codici: Ambrosianus D 525 inf. (appartenente alla famiglia "W"), Ambrosianus I 61 inf. (appartenente alla famiglia "W"), Basilicanus A. 4, Basilicanus B. 50 (appartenente alla famiglia "W"), Cantabrigiensis Mm. 4. 28 (appartenente alla famiglia

\footnotetext{
${ }^{179}$ Sul codice, cf. Degórski, Przekaz tacińskich rękopisów, p. 15, 71, 143, 159-160, 162, 227.

${ }^{180}$ Sul codice, cf. ibidem, p. 15, 72, 143, 148, 155, 159, 162, 227, 246-247, 342, 366, 390.

${ }^{181}$ Certo: "multa <allia>".

${ }^{182}$ Cf. Hieronymus, Vita Sancti Pauli 3, ed. Degórski, p. 81.

${ }^{183}$ Cf. Decalogus de beato Paulo 9.

${ }^{184}$ L'aggiunta $<$ nesciebat $>$ fu cancellata (sottolineata) probabilmente dalla prima mano.

${ }^{185}$ Cf. PL 23, 17-28.

${ }^{186}$ Cf. Hieronymus, Vita Sancti Pauli 9, ed. Degórski, p. 125.

${ }^{187}$ Cf. Decalogus de beato Paulo 13.
} 
“E”), e nei codici: Casanatensis Codex 253, 726 (appartenenti alla famiglia "I"), 1898 (appartenente alla famiglia "Q"), Erlangensis 161 (appartenente alla famiglia "E"), Iesuiticus 973 (appartenente alla famiglia "I"), Lateranensis Codex A. 79 (non classificabile), Monacensis 17137 (appartenente alla famiglia "E"), Novariensis LXI (9) (appartenente alla famiglia "W"), Remensis 1390 (appartenente alla famiglia "E”), Vallicellanus Codex C. 55 (appartenente alla famiglia "Q"), Vallicellanus Tomus II (appartenente alla famiglia "W"), Vallicellanus Tomus XII (appartenente alla famiglia "W"), Vaticanus Barberinianus Lat. 605 (appartenente alla famiglia "Q"), Vaticanus Barberinianus Lat. 621, e nei codici: Vaticanus Lat. 349, 358, 363 (appartenenti alla famiglia "I"), 374 (appartenente alla famiglia "W"), 1188 (unisce le famiglie "Q" e "W"), 1196 (appartenente alla famiglia "W"), 1199, 1200 (appartenenti alla famiglia "Q"), 1201, 5257 (non classificabile), $5411^{188}$ (non classificabile), 6933 (appartenente alla famiglia "Q"), Vaticanus Ottobonianus Lat. 120 (appartenente alla famiglia "D"), Vaticanus Palatinus Lat. 179 (appartenente alla famiglia "I"), Vaticanus Rossianus Lat. 287 (appartenente alla famiglia "Q"), Vaticanus Urbinas Lat. 48 (appartenente alla famiglia "Q"), Vaticanus Urbinas Lat. 51 (appartenente alla famiglia "Q"), Vaticanus Urbinas Lat. 1503. L'aggiunta <et> è presente inoltre nel Vallicellanus Tomus V (appartenente alla famiglia "I"), ma venne appostata da una seconda mano.

2. Le aggiunte presenti esclusivamente nel Decalogus

1) Dopo le parole "et Ioanne"189 nel Decalogus leggiamo: "et Ioanne $<$ baptista $>$ "190. 2) Dopo la parola "Alii" (tralasciando, quindi: "autem, in quam opinionem uulgus omne consentit"191) nel Decalogus leggiamo: " $<$ (vt Iheronymus) $>$ "192. 3) Al testo: "non nominis"193 il Decalogus aggiunge: " $<$ sed $>$ non nominis" 194 . L'aggiunta non si trova in alcun codice. Anziché $<$ sed $>$ troviamo <quod> nei codici: Parisinus Lat. 11749, Vaticanus Ottobonianus Lat. 120 (appartenenti alla famiglia “D”). L'aggiunta <quod> si trova anche nel $\mathrm{Wi}$ gorniensis $\mathrm{F} 48$, ma fu aggiunta a secunda manu super rasuram. Il Wigorniensis F 48 appartiene alla famiglia "L". 4) Al testo: "Perseuerantem in fide"195 il Decalogus aggiunge: "Perseuerantem < vnum> in fide"196. 5) Al testo: "Quid ageret miles Christi, quo"197 il Decalogus aggiunge: "Quid ageret miles Christi,

\footnotetext{
${ }^{188}$ Questo codicie, anche se aggiunge la congiunzione $<$ et $>$, tralascia la parola "unde".

${ }^{189}$ Cf. Hieronymus, Vita Sancti Pauli 1, ed. Degórski, p. 67.

${ }^{190} \mathrm{Cf}$. Decalogus de beato Paulo 8.

${ }^{191}$ Cf. Hieronymus, Vita Sancti Pauli 1, ed. Degórski, p. 68.

${ }^{192}$ Cf. Decalogus de beato Paulo 8.

${ }^{193}$ Cf. Hieronymus, Vita Sancti Pauli 1, ed. Degórski, p. 69-70.

${ }^{194}$ Cf. Decalogus de beato Paulo 8.

${ }^{195}$ Cf. Hieronymus, Vita Sancti Pauli 3, ed. Degórski, p. 76.

${ }^{196}$ Cf. Decalogus de beato Paulo 9.

${ }^{197}$ Cf. Hieronymus, Vita Sancti Pauli 3, ed. Degórski, p. 80.
} 
$<$ aut> quo"198. L'aggiunta <aut> non si trova in alcun codice. Anziché <aut> troviamo <uel $>$ nel Cantabrigiensis 389 (appartenente alla famiglia "D") ed $<$ et> nei codici: Parisinus Lat. 11749 (appartenente alla famiglia "D"), Parisinus Lat. 16050 (appartenente alla famiglia “R”), Vaticanus Ottobonianus Lat. 120 (appartenente alla famiglia “D”). L'aggiunta $<$ et> si trova pure nella PL.

3. Alcune lacune che non sono presenti in alcun manoscritto della Vita Pauli

Il Decalogus tralascia: 1) "autem, in quam opinionem uulgus omne consentit"199, 2) La parola "fuit", presente nella Vita Pauli, ove il testo suona: "Quorum quia impudens mendacium fuit" ${ }^{200}$. Non possiamo classificare il Decalogus in alcuna delle famiglie della tradizione manoscritta della Vita S. Pauli. E frutto di una tardiva contaminazione.

Speriamo che la presente nostra ricerca, riguardante la Vita S. Pauli monachi Thebaei, possa contribuire ad una futura sempre migliore pubblicazione del testo critico di questa prima opera monastica stilata in latino e che, altresì, risulti utile per la classificazione dei nuovi codici manoscritti della Vita Pauli, che forse saranno scoperti da codicologi, paleografi od altri studiosi delle antichità cristiane.

THE MANUSCRIPT TRADITION OF THE VITA S. PAULI PRIMI EREMITAE AND THE MOST ANCIENT PRINTED EDITIONS

OF THIS WORK OF ST. JEROME

(Summary)

The article is meant to present - in the first part - the results of the author's research on the Manuscript Tradition of the Vita S. Pauli monachi Thebaei of St. Jerome and, in particular, what was surveyed through the continuation of the analysis by John Frank Cherf O.S.B. In the second part of the article the most ancient printed editions of the Vita S. Pauli monachi Thebaei of St. Jerome are reviewed.

\footnotetext{
${ }^{198}$ Cf. Decalogus de beato Paulo 9.

${ }^{199}$ Cf. Hieronymus, Vita Sancti Pauli 1, ed. Degórski, p. 68.

${ }^{200} \mathrm{Cf}$. ibidem, ed. Degórski, p. 71. Anziché "mendacium", nel Decalogus troviamo "mendatium".
} 


\section{PRZEKAZ RĘKOPISÓW VITA S. PAULI PRIMI EREMITAE I NAJSTARSZE WYDANIA DRUKIEM TEGO HIERONIMOWEGO DZIEŁA}

\section{(Streszczenie)}

Artykuł w pierwszej swej części ukazuje wyniki badań nad rękopiśmiennym przekazem Vita S. Pauli monachi Thebaei św. Hieronima, a w szczególności to, co autor artykułu w tym względzie ustalił, kontynuując dzieło rozpoczęte przez Johna Franka Cherfa O.S.B.

W drugiej natomiast części artykuł przedstawia i analizuje od strony kodykologicznej najstarsze wydania drukiem wyżej wspomnianego dzieła św. Hieronima.

Key words: The Vita S. Pauli monachi Thebaei of Saint Jerome, Monasticism, Palaeography, Codicology.

Parole chiave: La Vita S. Pauli monachi Thebaei di san Girolamo, monachesimo, paleografia, codicologia.

Słowa kluczowe: Vita S. Pauli monachi Thebaei św. Hieronima, monastycyzm, paleografia, kodykologia.

\section{BIBLIOGRAFIA}

Fonti

Hieronymus, Vita Sancti Pauli Primi Eremitae, CPL 617:

- Vita diui Pauli primi heremite. Matthias Milcher librarius Budensis [...] Venetiis anno virginei partus 1511, die primo Decembris. Impensis Matthie Milcher librarii Budensis, arte autem Jacobi Pentii de Leucho, Reprint der Originalausgabe von 1511 mit einer Einfürung von S. Świdziński, Archivum Ordinis Sancti Pauli Primi Eremitae II: Fontes, vol. 10, ed. S. Świdziński, Coesfeld 2009.

- Decalogus de beato Paulo primo heremita comportatus per reverendum patrem fratrem Gregorium de Gyengyes protunc priorem sancti Stephani Rotundi in Vrbe cum annotationibus in margine adiecti, Cracouie per Florianum Unglerum 1532, Archivum Ordinis Sancti Pauli Primi Eremitae II: Fontes, vol. 9, ed. S. Świdziński, Coesfeld 2008.

- $\quad$ ed. R [R. = B.] Degórski: Anhang $I$ [edizione critica e traduzione polacca della Vita S. Pauli Primi Eremitae di san Girolamo, contenuta in G. Gyengyes, Decalogus de beato Paulo primo heremita, Cracoviae 1532], in: Archivum Ordinis Sancti Pauli Primi Eremitae II: Fontes, vol. 9, ed. S. Świdziński, Coesfeld 2008, 170-187.

- ed. R [R. = B.] Degórski: Edizione della "Vita Sancti Pauli Primi Eremitae" di San Girolamo, Institutum Patristicum “Augustinianum”, I-VI, Roma 1987 (dattiloscritto).

- $\quad$ ed. R [R. = B.] Degórski: Hieronymus, Vita Sancti Pauli Primi Eremitae. Edizione critica della "Vita Sancti Pauli Primi Eremitae” di Girolamo, Institutum Patristicum “Augustinianum”, Roma 1987. 


\section{Studi}

Cherf J.F., The Latin Manuscript Tradition of the "Vita S. Pauli", in: Studies in the Text Tradition of. St. Jerome's "Vitae Patrum”, ed. W.A. Oldfather, Urbana 1943, 65-142.

Degórski B., Kodeks Basilicanus A. 6 (alias E) a rodzina “ $Q$ ” rękopiśmiennego przekazu "Vita Sancti Pauli Primi Eremitae” św. Hieronima, VoxP 31 (2011) t. 56, 485-493.

Degórski B., La famiglia “I” della tradizione manoscritta della "Vita S. Pauli Primi Eremitae” di San Girolamo, DPa 5 (1992) 5-24.

Degónski B., La famiglia " $Q$ " della tradizione manoscritta della "Vita S. Pauli Primi Eremitae" di San Girolamo, DPa 5 (1992) 26-45.

Degórski B., La famiglia " $S$ " della tradizione manoscritta della "Vita Sancti Pauli Primi Eremitae” di San Girolamo, DPa 6 (1993) 16-41.

DegóRsKi B., La tecnica di preparazione di un'edizione critica. Un caso esemplativo: la familia " $Q$ " della tradizione manoscritta della "Vita S. Pauli Primi Eremitae" di san Girolamo, in: Sanctitatis Causae. Motivi di santità e Cause di canonizzazione di alcuni maestri medioevali. In ricordo di Padre Louis-Jacques Bataillon, O.P., ed. M.M. Rossi - T. Rossi, Studia Pontificiae Universitatis a S. Thoma Aquinate in Urbe. Studi 2008. Nuova serie 12, Roma 2009, 11-43.

Degórski B., Przekaz łacińskich rękopisów „, Vita S. Pauli Primi Eremitae” św. Hieronima, Lublin 2000.

Degórski B., Stan badań nad "Vita S. Pauli Primi Eremitae” św. Hieronima, in: Archivum Ordinis Sancti Pauli Primi Eremitae I: Dissertationes, vol. 5, ed. S. Świdziński Coesfeld 2010, 161-222.

DegóRski B., Un tardivo manoscritto di Cracovia contenente la "Vita Sancti Pauli Primi Eremitae" di San Girolamo, VoxP 8 (1988) t. 14, 429-434.

Degórski B., Untersuchungen zu den Handschriften und Drucken über die "Vita S. Pauli Primi Eremitae" vom hl. Hieronymus, in: Ideologie des Paulinermönchtums Ende 15./ Anfang 16. Jahrhundert. Internationales Seminar im ehemaligen Paulinerkloster (1377-1786) Marianka bei Bratislava, vom 11.-15./16 Oktober 2010, Archivum Ordinis Sancti Pauli Primi Eremitae I: Dissertationes, vol. 6/1, ed. L. Weinrich - S. Świdziński, Coesfeld 2012, 8-15.

Studies in the Text Tradition of. St. Jerome's “Vitae Patrum”, ed. W.A. Oldfather, Urbana 1943. 
